\title{
Identification of exosomal miRNAs associated with the anthracycline-induced liver injury in postoperative breast cancer patients by small RNA sequencing
}

\author{
Yue Zhang ${ }^{1}$, Di Wang ${ }^{2}$, Di Shen ${ }^{1}$, Yang Luo ${ }^{\text {Corresp., }}{ }^{3}$, Yi-Qun Che ${ }^{\text {Corresp. } 1}$ \\ 1 Department of Clinical Laboratory, National Cancer Center/ National Clinical Research Center for Cancer/ Cancer Hospital, Chinese Academy of Medical \\ Sciences and Peking Union Medical College, Beijing, P.R.China \\ 2 State Key Lab of Molecular Oncology, National Cancer Center/ National Clinical Research Center for Cancer/ Cancer Hospital, Chinese Academy of \\ Medical Sciences and Peking Union Medical College, Beijing, P.R.China \\ 3 Department of Medical Oncology, National Cancer Center/ National Clinical Research Center for Cancer/ Cancer Hospital, Chinese Academy of Medical \\ Sciences and Peking Union Medical College, Beijing, P.R.China \\ Corresponding Authors: Yang Luo, Yi-Qun Che \\ Email address: luoyang@cicams.ac.cn, cyq@cicams.ac.cn
}

Background . Anthracycline-induced liver injury (AILI) is one of the serious complications of anthracycline-based adjuvant chemotherapy for postoperative breast cancer patients. Exosomal miRNAs, as signaling molecules in intercellular communication, play the essential roles in drug-induced liver injury (DILI). However, the expression profiles of them in patients with AILI remains unknown.

Methods. Seven post-chemotherapy patients were recruited in this study. After isolated plasma-derived exosomes, small RNA sequencing revealed exosomal miRNA profiles and differentially expressed miRNAs (DE-miRNAs) were identified between the liver injury group and non-liver injury group. miRTarBase and miRDB were used to predict the potential target genes of DE-miRNAs. DILI-related genes were downloaded from the CTD Database. The intersection of predicted genes and DILI-related genes were identified as the AILI-related target genes of the DE-miRNAs. GO annotation and KEGG pathway enrichment analysis were performed by the DAVID database. Furthermore, the protein-protein interaction (PPI) network was established by STRING database and essential exosomal miRNAs were identified via Cytoscape software. Results. A total of 30 DE-miRNAs and 79 AlLI-related target genes were identified. AILI-related target genes of the DE-miRNAs are significantly enriched in NOD-like receptor signaling pathway, HIF-1 signaling pathway and FoxO signaling pathway. Then, the hub genes were screened and we discovered that IL- 6 and SOD2 are the most critical genes that may be involved in the development of AILI through the activation of immune response and the occurrence of oxidative stress, respectively. In addition, we found that miR-1-3p could potentially regulate most of the hub genes in the miRNA-hub gene network. Conclusion. We explored the potential functions of DE-miRNAs and suggested exosomal miR-1-3p might be the essential exosomal miRNA in the pathogenesis of AILI. Moreover, our study provided an experimental basis for experimental verification to reveal the actual function and mechanism of miRNAs in AlLI. 
1 Identification of exosomal miRNAs associated with

2 the anthracycline-induced liver injury in postoperative

3 breast cancer patients by small RNA sequencing

4

5 Yue Zhang 1 , Di Wang 2 , Di Shen ${ }^{1}$, Yang $\mathrm{Luo}^{3 *}$, Yi-Qun Che ${ }^{1 *}$

$6{ }^{1}$ Department of Clinical Laboratory, National Cancer Center/ National Clinical Research Center

7 for Cancer/ Cancer Hospital, Chinese Academy of Medical Sciences and Peking Union Medical

8 College, Beijing, P.R.China.

92 State Key Lab of Molecular Oncology, National Cancer Center/ National Clinical Research

10 Center for Cancer/ Cancer Hospital, Chinese Academy of Medical Sciences and Peking Union Medical College, Beijing, P.R.China.

${ }^{3}$ Department of Medical Oncology, National Cancer Center/ National Clinical Research Center for Cancer/ Cancer Hospital, Chinese Academy of Medical Sciences and Peking Union Medical College, Beijing, P.R.China.

${ }^{*}$ Correspondence: Yi-Qun Che, M.D. Professor, Department of Clinical Laboratory, National Cancer Center / National Clinical Research Center for Cancer/ Cancer Hospital, Chinese Academy of Medical Sciences and Peking Union Medical College. No.17 Panjiayuan Nanli, Chaoyang District, Beijing (100021), P.R. China. Tel./Fax: + 86-10-87788746. Email: cyq@cicams.ac.cn Yang Luo, M.D. Professor, Department of Medical Oncology, National Cancer Center/ National Clinical Research Center for Cancer/ Cancer Hospital, Chinese Academy of Medical Sciences and Peking Union Medical College. No.17 Panjiayuan Nanli, Chaoyang District, Beijing (100021), P.R. China. Tel./Fax: + 86-10-87788820. E-mail: luoyang@cicams.ac.cn 
34

35

36

37

38

39

40

41

42

43

44

45

46

47

48

49

50

51

52

53

54

55

56

57

58

59

60

61

62

63

64

65

66

67

68

69

70

71

72

73

\section{Abstract}

Background. Anthracycline-induced liver injury (AILI) is one of the serious complications of anthracycline-based adjuvant chemotherapy for postoperative breast cancer patients. Exosomal miRNAs, as signaling molecules in intercellular communication, play the essential roles in druginduced liver injury (DILI). However, the expression profiles of them in patients with AILI remains unknown.

Methods. Seven post-chemotherapy patients were recruited in this study. After isolated plasmaderived exosomes, small RNA sequencing revealed exosomal miRNA profiles and differentially expressed miRNAs (DE-miRNAs) were identified between the liver injury group and non-liver injury group. miRTarBase and miRDB were used to predict the potential target genes of DEmiRNAs. DILI-related genes were downloaded from the CTD Database. The intersection of predicted genes and DILI-related genes were identified as the AILI-related target genes of the DE-miRNAs. GO annotation and KEGG pathway enrichment analysis were performed by the DAVID database. Furthermore, the protein-protein interaction (PPI) network was established by STRING database and essential exosomal miRNAs were identified via Cytoscape software. Results. A total of 30 DE-miRNAs and 79 AILI-related target genes were identified. AILIrelated target genes of the DE-miRNAs are significantly enriched in NOD-like receptor signaling pathway, HIF-1 signaling pathway and FoxO signaling pathway. Then, the hub genes were screened and we discovered that IL-6 and SOD2 are the most critical genes that may be involved in the development of AILI through the activation of immune response and the occurrence of oxidative stress, respectively. In addition, we found that miR-1-3p could potentially regulate most of the hub genes in the miRNA-hub gene network.

Conclusion. We explored the potential functions of DE-miRNAs and suggested exosomal miR1-3p might be the essential exosomal miRNA in the pathogenesis of AILI. Moreover, our study provided an experimental basis for experimental verification to reveal the actual function and mechanism of miRNAs in AILI.

Keywords: Anthracycline-induced liver injury (AILI), exosomal, miRNAs, breast cancer

\section{Introduction}

The liver is the largest internal organ responsible for the selective uptake, metabolism, and excretion of endogenous and exogenous compounds, including drugs. The activity of uptake transporters that facilitate the accumulation of drugs in hepatocytes makes this organ susceptible to drug-induced liver injury (DILI) (Kolarić et al., 2019). The true incidence of DILI is difficult to determine due to its lack of standardized diagnostic criteria, delay in diagnosis and unpredictable nature, but the population incidence is likely on the rise. DILI represents a serious clinical problem and potentially fatal course (Hayashi \& Chalasani, 2015; Cano-Paniagua et al., 2019).

Breast cancer is the most frequently diagnosed cancer and the leading cause of cancer death in females worldwide (Siegel, Miller \& Jemal, 2019). Treatment with adjuvant chemotherapy is recommended for women with resected node-positive or high-risk node-negative breast cancer, 
74 and an anthracycline-based regimen is often included (Waks \& Winer, 2019). Anthracycline,

75

76

77

78

79

80

81

82

83

84

85

86

87

88

89

90

91

92

93

94

95

96

97

98

99

100

101

102

103

104

105

106

107

108

109

110

111

112

113 mainly including doxorubicin and epirubicin, is a class of antibiotics derived from the Streptomyces bacteria and has pleiotropic effects including free radical formation, topoisomerase II inhibition and altered mitochondrial function. However, it is associated with potential longterm effects such as chronic cardiotoxicity and liver injury (Wu et al., 2016; Joerger, 2016). The magnitude of anthracycline-induced liver injury (AILI) in breast cancer patients is rising as a result of the increasing number of long-term cancer survivors and because of the tendency to use higher doses of anthracyclines, as well as combined endocrine treatments with synergistic liver toxic effects. As the clinical standard, serum transaminases are generally used for evaluating liver injury (Kagawa et al., 2018). However, recent evidence suggests that the release of danger signals may occur in the absence of hepatocyte necrosis and that even minor serum ALT elevations that are often observed in a large percentage of patients treated with drugs reflect hepatocyte death (Mosedale et al., 2018). Early diagnosis of AILI is a crucial point for AILI prevention and treatment. Research is needed to understand the mechanisms for AILI, particularly involving its genetic basis.

Currently, exosomes have drawn considerable attention for providing sensitive and detailed insights into many diseases. Exosomes are small extracellular membrane-bound vesicles ranging from 20-150 nm secreted by a variety of cell types, which contain selective particles, including mRNA, miRNA, and proteins. The primary function of these vesicles is cell-cell communication in multiple pathophysiological processes (Barile \& Vassalli, 2017). MicroRNAs (miRNAs) are small non-coding RNA molecules that can regulate the gene expression post-transcriptionally. With the high stability and protection against RNase-mediated degradation in exosomes, miRNA are attractive candidates to contain valuable biomarkers or exhibit therapeutic effects on experimental and clinical conditions (Hammond, 2015). Previous researchers reported that exosome-associated biomarkers have higher specificity and sensitivity than transaminases, importantly, their serum levels appeared to rise earlier than serum transaminases levels (Masyuk, Masyuk \& LaRusso, 2013). Therefore, exosomal miRNAs are generally considered stable in peripheral blood and may serve as minimally invasive biomarkers for early detection and prognosis of DILI. Whereas, the role of exosomal miRNAs in AILI remains poorly understood. In the current study, small RNA sequencing was performed to detect exosomal miRNAs in the postoperative breast cancer patients after receiving anthracycline-based adjuvant chemotherapy and DE-miRNAs were identified between the non-liver injury group and liver injury group. Moreover, bioinformatics analysis revealed the potential functions of DE-miRNAs and recognized miR-1-3p is critical exosomal miRNAs and may be novel biomarkers for early detection, diagnosis, and treatment of AILI.

\section{Materials \& Methods}

\section{Study Participants}

We recruited 7 postoperative breast cancer patients at the National Cancer Center/Cancer Hospital, Chinese Academy of Medical Sciences (Beijing, China), from September 2017 to

Peer) reviewing PDF | (2019:09:41024:1:2:NEW 25 Feb 2020) 
114 January 2018. Patients with any of the following conditions or previous treatments were

115

116

117

118

119

120

121

122

123

124

125

126

127

128

129

130

131

132

133

134

135

136

137

138

139

140

141

142

143

144

145

146

147

148

149

150

151

152

153

ineligible: previous invasive breast cancer; non-breast cancer within 5 years before randomization, with the exception of carcinoma in situ of the cervix or colon, melanoma in situ, and skin basal-cell or squamous-cell carcinomas; any previous chemotherapy or radiotherapy for cancer; abnormal renal or hepatic function; and concurrent diseases interfering with planned laboratory tests, such as diabetes, hypertension, etc. Only those participants who received 4 cycles (every 3 weeks) of epirubicin or doxorubicin plus cyclophosphamide, followed 4 cycles (every 2 weeks) of paclitaxel were eligible for this study. The cumulative dose of epirubicin exceeded $320 \mathrm{mg} / \mathrm{m}^{2}$. Written informed consent was obtained from all patients before enrollment, and the study was approved by the institutional ethical committee. National Cancer

Center/Cancer Hospital, Chinese Academy of Medical Sciences granted Ethical approval to carry out the study within its facilities (Ethical Application Ref: NCC1774).

\section{ALT detection and grouping of patients}

In order to assess the liver function after chemotherapy, approximately $3 \mathrm{ml}$ of blood from each patient was collected in SST serum separation tubes (Becton Dickinson) and centrifuged at 3000 $\times \mathrm{g}$ for $10 \mathrm{~min}$ at $4^{\circ} \mathrm{C}$. The serum of all patients was analyzed for ALT using a Cobas c501 analyzer (Roche Diagnostics, Germany), and kits were procured by Roche. The criterion of liver injury was set as ALT>40U/L (Yu et al., 2017).

\section{Sample collection and exosomes extraction}

All of the whole blood samples from individuals were obtained and collected in K2EDTA tubes (Becton Dickinson) using standard venipuncture procedures. After centrifugation at 3,000 $\times \mathrm{g}$ for 15 min at $4{ }^{\circ} \mathrm{C}$, the plasma was aspirated into micro tubes and stored at $-80^{\circ} \mathrm{C}$ fridge before use. plasma-derived exosomes were isolated by differential centrifugation according to the protocol previously described (Théry et al., 2006). After thawing at $37^{\circ} \mathrm{C}$, plasma was centrifugated at $3,000 \mathrm{~g}$ at $4{ }^{\circ} \mathrm{C}$ for $15 \mathrm{~min}$ to remove cell debris. The supernatant was diluted by a seven-fold volume of phosphate-buffered saline (PBS), centrifuged at $13,000 \mathrm{~g}$ at $4{ }^{\circ} \mathrm{C}$ for $30 \mathrm{~min}$, and filtered through a $0.22 \mu \mathrm{m}$ filter to eliminate large particles. Then, the supernatant was ultracentrifuged using a P50A72-986 rotor (CP100NX; Hitachi, Brea, CA, USA) at 100,000g at $4{ }^{\circ} \mathrm{C}$ for $2 \mathrm{~h}$ to pellet the exosomes. The pellet was resuspended in PBS and centrifuged again at $100,000 \mathrm{~g}$ at $4{ }^{\circ} \mathrm{C}$ for $2 \mathrm{~h}$. After PBS washing, exosome pellets were resuspended in $100 \mu \mathrm{PBS}$ for TEM, NTA, and further research.

\section{Exosomes characterize validation}

The exosome pellets were observed and photographed by a transmission electron microscope (JEOL-JEM1400, Tokyo, Japan). Briefly, $10 \mu$ l exosomes solution was dropped onto a square copper grid and incubated for $10 \mathrm{~min}$ at room temperature. After washing with sterile distilled water, the exosomes were negatively stained with uranyl oxalate solution for $1 \mathrm{~min}$. Then, the sample was dried under incandescent light for $2 \mathrm{~min}$ before viewing.

PeerJ reviewing PDF | (2019:09:41024:1:2:NEW 25 Feb 2020) 
154 For the purpose of measuring size distribution and concentration, nanoparticle tracking analysis

155 (NTA) was performed using the ZetaView PMX 110 (Particle Metrix, Meerbusch, Germany) and 156 corresponding software NTA software (ZetaView 8.02.28) as recommended by the company.

157 Western blot analysis was performed to detect exosomal surface markers. Briefly, the exosome

158 supernatant was denatured in sodium dodecyl sulfonate (SDS) buffer and then incubated with

159 CD63, TSG101, Alix and Calnexin. The proteins were finally visualized with a Tanon4600

160 Automatic chemiluminescence image analysis system (Tanon, Shanghai, China).

161

162

\section{MicroRNA sequencing and data analysis}

163 Total RNA in plasma-derived exosomes was extracted and purified using a miRNeasy ${ }^{\circledR}$ Mini kit 164 (Qiagen, Cat. No. 217004) according to the manufacturer's instruction. The degradation and contamination of RNA were monitored on $1.5 \%$ agarose gels. The concentration and purity of

166

167

168

169

170

171

172

173

174

175

176

177

178

179

180

181

182

183

184

185

186

187

188

189

190

191

192 RNA were assessed using NanoDrop 2000 Spectrophotometer (Thermo Fisher Scientific, Wilmington, DE) and RNA Nano 6000 Assay Kit of the Agilent Bioanalyzer 2100 System (Agilent Technologies, CA, USA). The concentration of RNA is more than $500 \mathrm{pg} / \mathrm{ul}$ in all samples. Library preparation and sequencing of miRNA were performed by Beijing ECHO BIOTECH Co. Ltd. In brief, after ligated with 3' and 5' adaptors, small RNAs were reversetranscribed to cDNA and amplified by PCR. The products from PCR were sequenced on an Illumina HiSeq 2500 platform (Data S1). Considering that some miRNAs have little or no expression in some samples, only those with raw count value $>3$ in more than $75 \%$ of samples were retained for further analysis. The fold-change and P-value were calculated based on the miRNA counts using the package edgeR (Robinson, McCarthy \& Smyth, 2010) of R and differentially expressed miRNAs were identified between groups of interest. P-value $<0.05$ and $\mid \log 2 \mathrm{FC}$ (fold change) $\mid>1$ were set as the cut-off criteria to screen out DE-miRNAs (Code S1).

\section{Identification of AILI-related target genes}

miRTarBase (Chou et al., 2018), an experimentally supported microRNA-target interactions database, was applied to predict the targets of the known miRNAs. The target genes of novel miRNAs were predicted using miRDB (Liu \& Wang, 2019) and the screening criterion was set as score $\geq 80$. Besides, we searched "drug-induced liver injury" in the Comparative Toxicogenomics Database (CTD, revision 15982) (Davis et al., 2019) and got the DILI-related genes (marker, mechanism or therapeutic genes of DILI). To narrow the research scope for further research, the intersection of predicted genes and DILI-related genes were identified as the AILI-related target genes of the DE-miRNAs.

\section{Functional Annotation and Pathway Enrichment Analysis}

Gene Ontology (GO) annotation and Kyoto Encyclopedia of Genes and Genomes (KEGG) pathway enrichment analysis for AILI-related target genes of the DE-miRNAs were performed using the database for annotation, visualization and integrated discovery (DAVID 6.8) (Huang, 
193 Sherman \& Lempicki, 2009). Only the GO terms and KEGG pathways with $\mathrm{P}<0.05$ were

194

195

196

197

198

199

200

201

202

203

204

205

206

207

208

209

210

211

212

213

214

215

216

217

218

219

220

221

222

223

224

225

226

227

228

229

230

231

232

considered statistically significant.

\section{PPI network and miRNA-hub gene network construction}

To better understand the interaction among the AILI-related target genes, the protein-protein interaction (PPI) network was generated using the STRING database (Szklarczyk et al., 2015) and only the interactions with a combined score $>0.4$ were considered significant. Then, the PPI network was visualized and analyzed using Cytoscape software (version 3.7.1) (Shannon et al., 2003). The Cytohubba plug-in of Cytoscape (version 0.1) (Chin et al., 2014) was used to identify important hub genes of the entire network. The maximal clique centrality (MCC) method was used to identifying hub objects in this study because this method could capture more essential proteins in the top-ranked list in both high degree and low degree proteins. After that, miRNAhub gene networks were constructed by Cytoscape software.

\section{Results}

\section{Serum biochemical changes in patients}

Seven postoperative breast cancer patients who underwent chemotherapy were recruited in our study. To assess liver function, serum ALT level was measured in all individuals before and after chemotherapy. According to the results of the serum ALT level (Fig.1, Data S1), seven patients were divided into two groups: the non-liver injury group $(n=2)$ and the liver injury group $(n=5)$.

\section{Verification of exosomes}

After isolated from the plasma, exosomes were verified by TEM, NTA and western blot. The morphology of exosomes was visualized under a transmission electron microscope (TEM), and we found homogeneous cup-shaped vesicles with lipid bilayer membranes (Fig.2A). We then performed a nanoparticle tracking analysis (NTA) to measure the size distribution of exosomes, and the results revealed the most widely distributed particle diameter was $122.9 \mathrm{~nm}$ (Fig.2B). Additionally, exosomal surface markers were detected using western blot analysis. As shown in Fig.2C and Data S2, exosome-positive markers (Alix, TSG101, and CD63) were identified, while the negative marker for exosomes (Calnexin) was absent in the isolated exosomes. These results demonstrated that exosomes were successfully isolated from the plasma.

\section{Identification of DE-miRNAs and AILI-related target genes}

We performed high throughput sequencing to detect the miRNA expression of exosomes and screened DE-miRNAs between non-liver injury group and liver injury group using edgeR with $\mathrm{P}<0.05$ and $\left|\log _{2} \mathrm{FC}\right|>1$. The results showed that a total of 2,031 genes had been detected, including 1576 known miRNAs and 455 novel miRNAs. There are 30 DE-miRNAs were screened, of which 16 miRNAs (1 novel miRNA and 15 known miRNAs) were upregulated, and 14 miRNAs ( 1 novel miRNA and 13 known miRNAs) were downregulated. The volcano plots and heatmap of DE-miRNAs between the two groups are shown in Fig.3. Then, we employed 
233 two miRNA-target interactions database, miRTarBase and miRDB, to predict the target genes of

234 known and novel DE-miRNAs, respectively. As a result, 3823 predicted targets from

235 miRTarbase and miRDB were identified as the target genes of 30 DE-miRNAs. Genes of DILI

236 were downloaded from the CTD Database. A total of 433 DILI-related genes were recorded. To

237 narrow the research scope, the intersection of predicted genes and DILI-related genes were

238 identified as the AILI-related target genes of the DE-miRNAs. Finally, 79 AILI-related target

239 genes were screened, including 55 and 36 targets for upregulated and downregulated DE-

240 miRNA, respectively (Fig.3C).

241

242 Functional analysis of the AILI-related target genes

243 To evaluate the biological functions of these screened AILI-related target genes, GO annotation

244 and KEGG pathway enrichment analysis were performed by using DAVID (Table S1).

245 Three functional categories were selected in GO annotation, including biological process (BP),

246 cellular component (CC), and molecular function (MF). In the BP category, AILI-related target

247 genes of upregulated miRNAs were significantly enriched in response to drug, positive

248 regulation of MAPK cascade, and response to amino acid. In the CC category, these genes were

249 significantly enriched in extracellular exosome, extracellular space, and cell-cell adherens

250 junction. In addition, these genes were mainly enriched in growth factor activity, cadherin

251 binding involved in cell-cell adhesion, and oxidoreductase activity in the MF category (Fig.4A).

252 Besides, AILI-related target genes of downregulated miRNAs were significantly enriched in

253 cellular response to vascular endothelial growth factor stimulus, coronary vein morphogenesis,

254 and response to lipopolysaccharide in the BP category. In the CC category, they were mainly

255 enriched in extracellular exosome, myelin sheath, and platelet alpha granule lumen. Moreover,

256 these genes were significantly enriched in enzyme binding, platelet-derived growth factor

257 receptor binding, and chemoattractant activity in the MF category (Fig.4B).

258 KEGG pathway analysis was further conducted for AILI-related target genes of DE-miRNAs.

259 The most significantly enriched pathways of upregulated miRNAs included adherens junction,

260 rheumatoid arthritis, and HIF-1 signaling pathway (Fig.4A). The AILI-related target genes of

261 downregulated miRNAs were enriched in some terms but none of them are significant (Fig.4B).

262

263 PPI network and miRNA-hub gene network construction

264 The PPI network of AILI-related target genes was constructed using the STRING database

265 (Fig.5A). Then, we applied Cytohubba plug-in of Cytoscape to screen out the top 10 hub genes

266 of the network using the MCC method (Table 1). In the PPI network, the hub genes were IL6,

267 VEGFA, CCL2, HMOX1, IGF1, ACTB, CXCL1, SOD2, NOTCH1, and ARG1. Subsequently,

268 the miRNA-hub gene network was constructed by Cytoscape software. As shown in Fig.5B, the

269 hub target genes are regulated by 6 DE-miRNAs and miR-1-3p regulates the most hub

270 genes $(\mathrm{n}=8)$. Our results suggest that miR-1-3p may play an important role in AILI.

271 


\section{Discussion}

273 Recently, researchers and clinicians have found that exosomal miRNAs are more likely to be

274 applied as promising minimally invasive biomarkers in drug-induced liver injury. Motawi TK et

275 al. reported that exosomal miR-122a-5p exhibited higher diagnostic performance with a broader

276 diagnostic time window and an earlier diagnostic potential than its corresponding total serum

277 levels in thioacetamide (TAA)-induced acute liver injury (Motawi et al., 2018). Similarly, in the

278 absence of overt hepatocellular toxicity, significant elevation of exosomal miR-122 was

279 observed in primary human hepatocytes at subtoxic acetaminophen (APAP) concentration for

280 24h (Holman et al., 2016).

281 To our knowledge, this study is the first to investigate the expression profiling of serum

282 exosomal miRNAs in breast cancer patients with AILI. According to the detection results of

283 alanine aminotransferase in patients after chemotherapy, we found that anthracycline-based

284 adjuvant chemotherapy may induce liver injury. Then, we performed bioinformatics to analyze

285 the miRNA expression profile in exosomes and found the potential mechanism of AILI.

286 The KEGG pathway enrichment analysis was performed on the AILI-related target genes. It's

287 worth noting that some terms have been demonstrated to be associated with drug-induced liver

288 injury. FoxO signaling pathway involved in oxidative stress-induced apoptosis and there is

289 evidence that FoxO3 serves a proapoptotic role in hepatocellular apoptosis under oxidative stress

290 (Tao et al., 2013). Activated HIF-1 signaling pathway promoted hepatocellular necrosis at the

291 early time of APAP toxicity (Sparkenbaugh et al., 2011). In addition, Heatstroke-induced

292 hepatocyte exosomes aggravate liver damage and inflammation by activating the NOD-like

293 receptor signaling pathway in mice, which has been validated in the study of Li et al. (Li et al.,

294 2019). Therefore, these pathways are most likely involved in the pathogenesis and progression of

295 AILI.

296 Based on the STRING database and Cytohubba plug-in of Cytoscape, significant hub genes have

297

298

299

300

301

302

303

304 been screened out from the PPI network. Interleukin 6 (IL6), which encodes a cytokine involved in inflammation, is the hub gene with the highest score in the network. Previous studies have shown that IL-6 can promote liver regeneration and repair, but it can also make the liver sensitive to injury, stimulate hepatocyte apoptosis, induce insulin resistance, and participate in the occurrence and development of non-alcoholic steatohepatitis (NASH) (Braunersreuther et al., 2012). In the miRNA-hub gene network, superoxide dismutase 2 (SOD2) has the most connections to the DE-miRNAs and three of the four DE-miRNAs interacting with SOD2 are downregulated. SOD2 is an important antioxidant defense against oxidative stress, catalyzing the dismutation of superoxide (O2-) into oxygen and hydrogen peroxide(Miao \& St. Clair, 2009). Li et al. reported that SOD2 protects the liver from reperfusion injury following severe shock (Li et al., 2015). Therefore, the activation of immune response and the occurrence of oxidative stress may play a key role in the development of AILI.

310 The miRNA-hub gene network also helps us to find the essential exosomal miRNAs in the development of liver injury. We discovered that most of the hub genes $(n=8)$ could be potentially regulated by miR-1-3p. The previous study revealed that miR-1-3p significantly increased in 
312 APAP- and TAA-induced hepatocellular injury models. Besides, miR-1-3p also increased in the 313 early stages of $\alpha$-naphthylisothiocyanate- and 4,4'-methylenedianiline-induced cholestasis

314 models ( Kagawa et al., 2018). Additionally, researchers have found that the expression level of 315 miR-503-5p was significantly decreased with chronic low-dose exposure to microcystin-LR in 316 mice liver tissues (Xu et al., 2018).

317 There are some limitations to our study. First of all, due to the strict enrollment conditions and 318 the low number of patients with AILI, only seven patients were recruited in this study.It is 319 necessary to recruit more patients to get a more solid conclusion. Secondly, considering that the 320 verification experiment requires a large amount of time, we have not verified the results of bioinformatics analysis. AILI is highly correlated with patients' quality of life, but there are few studies on it. Through miRNA sequencing and bioinformatics analysis, we got some valuable results and shared them as soon as possible to accelerate AILI research.

\section{Conclusions}

Taken together, we found that ALT was significantly increased in postoperative breast cancer

327

328

329

330

331

332

333

334

335

336

337

338

339

340

341

342

343

344

345

346

347

348

349

350

351

352

353

patients after using the regimens containing anthracycline. Through the assistance of high throughput sequencing and bioinformatics analysis, we identified the potential regulatory functions of differentially expressed exosomal miRNAs and screened out the essential exosomal miRNAs in the development of AILI. Moreover, our study provides an experimental basis for experimental verification to reveal the actual function and mechanism of miRNAs in AILI.

\section{Funding}

This work was supported by the Chinese Academy of Medical Sciences Innovation Fund for Medical Sciences (Grant No.2017-I2M-3-012 \& No.2017-I2M-1-013).

\section{Disclosure}

The authors report no conflicts of interest in this work.

\section{References}

Barile L, Vassalli G. 2017. Exosomes: Therapy delivery tools and biomarkers of diseases. Pharmacology and Therapeutics 174:63-78. DOI: 10.1016/j.pharmthera.2017.02.020.

Braunersreuther V, Viviani GL, Mach F, Montecucco F. 2012. Role of cytokines and chemokines in non-alcoholic fatty liver disease. World Journal of Gastroenterology 18:727-735. DOI: 10.3748/wjg.v18.i8.727.

Cano-Paniagua A, Amariles P, Angulo N, Restrepo-Garay M. 2019. Epidemiology of druginduced liver injury in a University Hospital from Colombia: Updated RUCAM being used for prospective causality assessment. Annals of hepatology 18:501-507. DOI: 10.1016/j.aohep.2018.11.008.

Chin C-H, Chen S-H, Wu H-H, Ho C-W, Ko M-T, Lin C-Y. 2014. cytoHubba: identifying hub objects and sub-networks from complex interactome. BMC systems biology 8 Suppl 4:S11. DOI: 10.1186/1752-0509-8-S4-S11.

Chou C-H, Shrestha S, Yang C-D, Chang N-W, Lin Y-L, Liao K-W, Huang W-C, Sun T-H, Tu S-J, Lee W-H, Chiew M-Y, Tai C-S, Wei T-Y, Tsai T-R, Huang H-T, Wang C-Y, Wu H-Y, 
366

367

368

369

370

371

372

373

374

375

376

377

378

379

380

381

382

383

384

385

386

387

388

389

390

391

392

393

394

395

396

397

398

399

Ho S-Y, Chen P-R, Chuang C-H, Hsieh P-J, Wu Y-S, Chen W-L, Li M-J, Wu Y-C, Huang X-Y, Ng FL, Buddhakosai W, Huang P-C, Lan K-C, Huang C-Y, Weng S-L, Cheng Y-N, Liang C, Hsu W-L, Huang H-D. 2018. miRTarBase update 2018: a resource for experimentally validated microRNA-target interactions. Nucleic acids research 46:D296D302. DOI: 10.1093/nar/gkx1067.

Davis AP, Grondin CJ, Johnson RJ, Sciaky D, McMorran R, Wiegers J, Wiegers TC, Mattingly CJ. 2019. The Comparative Toxicogenomics Database: update 2019. Nucleic acids research 47:D948-D954. DOI: 10.1093/nar/gky868.

Hammond SM. 2015. An overview of microRNAs. Advanced drug delivery reviews 87:3-14. DOI: $10.1016 /$ j.addr.2015.05.001.

Hayashi PH, Chalasani NP. 2015. Increasing impact of drug-induced liver injury. Clinical Liver Disease 5:136-138. DOI: 10.1002/cld.469.

Holman NS, Mosedale M, Wolf KK, LeCluyse EL, Watkins PB. 2016. Subtoxic Alterations in Hepatocyte-Derived Exosomes: An Early Step in Drug-Induced Liver Injury? Toxicological sciences : an official journal of the Society of Toxicology 151:365-75. DOI: 10.1093/toxsci/kfw047.

Huang DW, Sherman BT, Lempicki RA. 2009. Systematic and integrative analysis of large gene lists using DAVID bioinformatics resources. Nature protocols 4:44-57. DOI: 10.1038/nprot.2008.211.

Joerger M. 2016. Treatment regimens of classical and newer taxanes. Cancer chemotherapy and pharmacology 77:221-33. DOI: 10.1007/s00280-015-2893-6.

Kagawa T, Shirai Y, Oda S, Yokoi T. 2018. Identification of Specific MicroRNA Biomarkers in Early Stages of Hepatocellular Injury, Cholestasis, and Steatosis in Rats. Toxicological sciences : an official journal of the Society of Toxicology 166:228-239. DOI: $10.1093 /$ toxsci/kfy200.

Kolarić TO, Ninčević V, Smolić R, Smolić M, Wu GY. 2019. Mechanisms of Hepatic Cholestatic Drug Injury. Journal of clinical and translational hepatology 7:86-92. DOI: 10.14218/JCTH.2018.00042.

Li P, Wang X, Zhao M, Song R, Zhao KS. 2015. Polydatin protects hepatocytes against mitochondrial injury in acute severe hemorrhagic shock via SIRT1-SOD2 pathway. Expert Opinion on Therapeutic Targets 19:997-1010. DOI: 10.1517/14728222.2015.1054806.

Li Y, Zhu X, Zhang M, Tong H, Su L. 2019. Heatstroke-induced hepatocyte exosomes promote liver injury by activating the NOD-like receptor signaling pathway in mice. PeerJ 2019. DOI: $10.7717 /$ peerj.8216.

Liu W, Wang X. 2019. Prediction of functional microRNA targets by integrative modeling of microRNA binding and target expression data. Genome Biology 2019;20(1):18. doi: 10.1186/s13059-019-1629-z.

Masyuk AI, Masyuk T V., LaRusso NF. 2013. Exosomes in the pathogenesis, diagnostics and therapeutics of liver diseases. Journal of Hepatology 59:621-625. DOI: 10.1016/j.jhep.2013.03.028.

Miao L, St. Clair DK. 2009. Regulation of superoxide dismutase genes: Implications in disease. Free Radical Biology and Medicine 47:344-356. DOI: 10.1016/j.freeradbiomed.2009.05.018.

Mosedale M, Eaddy JS, Trask OJ, Holman NS, Wolf KK, LeCluyse E, Ware BR, Khetani SR, Lu J, Brock WJ, Roth SE, Watkins PB. 2018. miR-122 Release in Exosomes Precedes Overt Tolvaptan-Induced Necrosis in a Primary Human Hepatocyte Micropatterned

Peer) reviewing PDF | (2019:09:41024:1:2:NEW 25 Feb 2020) 
400

401

402

403

404

405

406

407

408

409

410

411

412

413

414

415

416

417

418

419

420

421

422

423

424

425

426

427

428

429

430

431

432

433

434

435

436

437

438

439

440

441

442

443

444

445

Coculture Model. Toxicological sciences : an official journal of the Society of Toxicology 161:149-158. DOI: 10.1093/toxsci/kfx206.

Motawi TK, Mohamed MR, Shahin NN, Ali MAM, Azzam MA. 2018. Time-course expression profile and diagnostic potential of a miRNA panel in exosomes and total serum in acute liver injury. The International Journal of Biochemistry \& Cell Biology 100:11-21. DOI: 10.1016/j.biocel.2018.05.002.

Robinson MD, McCarthy DJ, Smyth GK. 2010. edgeR: a Bioconductor package for differential expression analysis of digital gene expression data. Bioinformatics 26:139-140. DOI: 10.1093/bioinformatics/btp616.

Shannon P, Markiel A, Ozier O, Baliga NS, Wang JT, Ramage D, Amin N, Schwikowski B, Ideker T. 2003. Cytoscape: a software environment for integrated models of biomolecular interaction networks. Genome research 13:2498-504. DOI: 10.1101/gr.1239303.

Siegel RL, Miller KD, Jemal A. 2019. Cancer statistics, 2019. CA: a cancer journal for clinicians 69:7-34. DOI: 10.3322/caac.21551.

Sparkenbaugh EM, Saini Y, Greenwood KK, LaPres JJ, Luyendyk JP, Copple BL, Maddox JF, Ganey PE, Roth RA. 2011. The Role of Hypoxia-Inducible Factor-1 in Acetaminophen Hepatotoxicity. Journal of Pharmacology and Experimental Therapeutics 338:492-502. DOI: 10.1124 jpet.111.180521.

Szklarczyk D, Franceschini A, Wyder S, Forslund K, Heller D, Huerta-Cepas J, Simonovic M, Roth A, Santos A, Tsafou KP, Kuhn M, Bork P, Jensen LJ, von Mering C. 2015. STRING v10: protein-protein interaction networks, integrated over the tree of life. Nucleic acids research 43:D447-52. DOI: 10.1093/nar/gku1003.

Tao G-Z, Lehwald N, Jang KY, Baek J, Xu B, Omary MB, Sylvester KG. 2013. Wnt/ $\beta$-catenin signaling protects mouse liver against oxidative stress-induced apoptosis through the inhibition of forkhead transcription factor FoxO3. The Journal of biological chemistry 288:17214-24. DOI: 10.1074/jbc.M112.445965.

Théry C, Amigorena S, Raposo G, Clayton A. 2006. Isolation and Characterization of Exosomes from Cell Culture Supernatants and Biological Fluids. Current Protocols in Cell Biology 30:3.22.1-3.22.29. DOI: 10.1002/0471143030.cb0322s30.

Waks AG, Winer EP. 2019. Breast Cancer Treatment: A Review. JAMA 321:288-300. DOI: 10.1001/jama.2018.19323.

Wu J, Xue X, Zhang B, Jiang W, Cao H, Wang R, Sun D, Guo R. 2016. The protective effects of paeonol against epirubicin-induced hepatotoxicity in 4T1-tumor bearing mice via inhibition of the PI3K/Akt/NF-kB pathway. Chemico-biological interactions 244:1-8. DOI: 10.1016/j.cbi.2015.11.025.

Xu L, Li T, Ding W, Cao Y, Ge X, Wang Y. 2018. Combined seven miRNAs for early hepatocellular carcinoma detection with chronic low-dose exposure to microcystin-LR in mice. Science of the Total Environment 628-629:271-281. DOI: 10.1016/j.scitotenv.2018.02.021.

Yu Y-C, Mao Y-M, Chen C-W, Chen J-J, Chen J, Cong W-M, Ding Y, Duan Z-P, Fu Q-C, Guo X-Y, Hu P, Hu X-Q, Jia J-D, Lai R-T, Li D-L, Liu Y-X, Lu L-G, Ma S-W, Ma X, Nan YM, Ren H, Shen T, Wang H, Wang J-Y, Wang T-L, Wang X-J, Wei L, Xie Q, Xie W, Yang C-Q, Yang D-L, Yu Y-Y, Zeng M, Zhang L, Zhao X-Y, Zhuang H, Drug-induced Liver Injury (DILI) Study Group, Chinese Society of Hepatology (CSH), Chinese Medical Association (CMA). 2017. CSH guidelines for the diagnosis and treatment of drug-induced liver injury. Hepatology international 11:221-241. DOI: 10.1007/s12072-017-9793-2. 
Figure 1

Before-after plots demonstrate the changes of serum ALT levels in breast cancer patients during chemotherapy.

Serum transaminase levels were significantly increased in five of the patients, but not significantly in the other two.

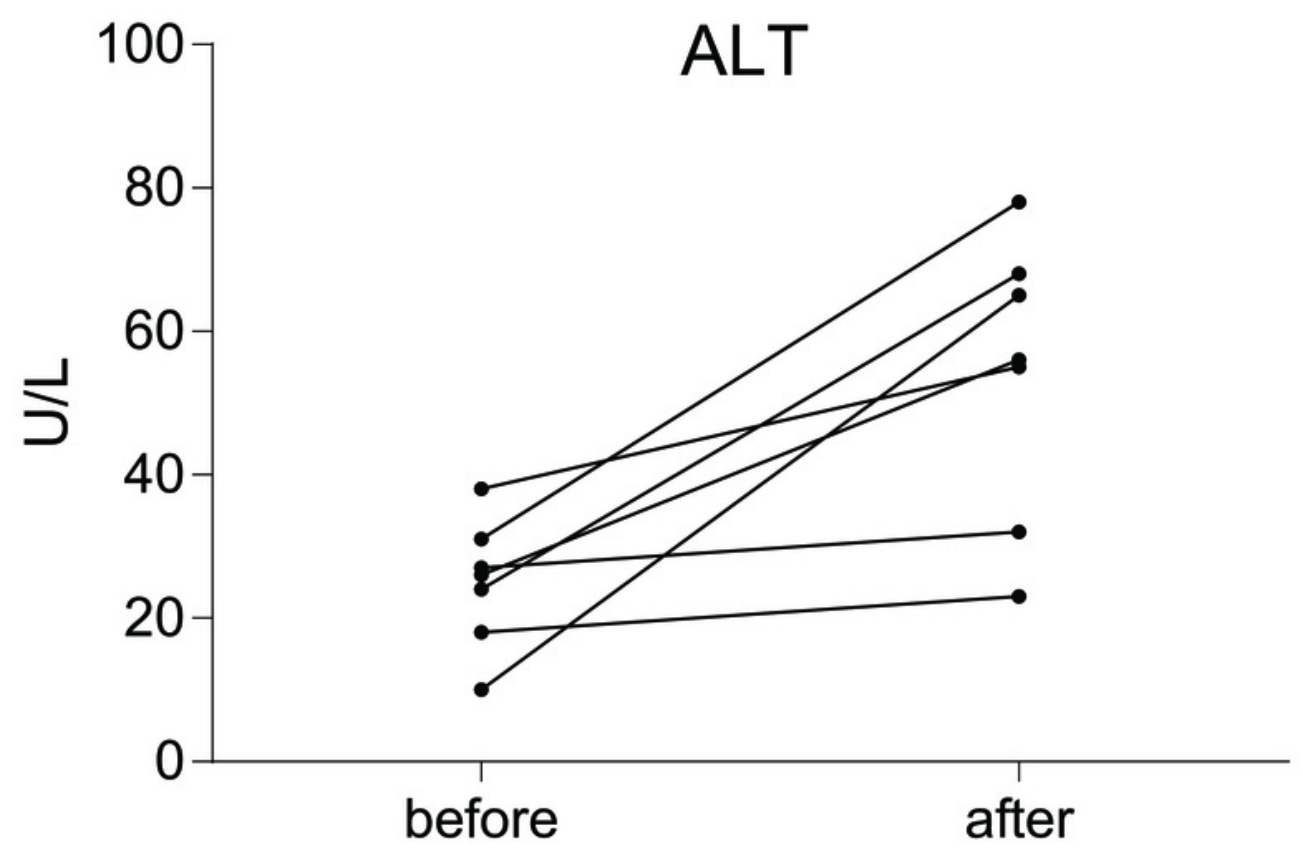


Figure 2

Characterization of exosomes isolated from plasma.

(A) Representative TEM images of exosomes isolated from plasma. Scale Bar=200 nm. (B) Nanoparticle tracking analysis revealed a size distribution of the exosomes. (C) Western Blot analysis for exosomes marker in exosomes and cell lysates.

A

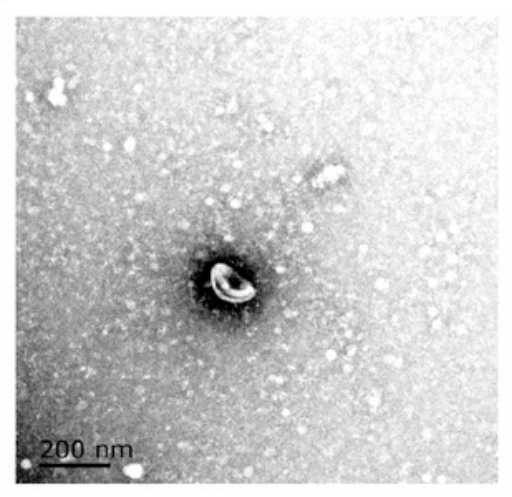

B

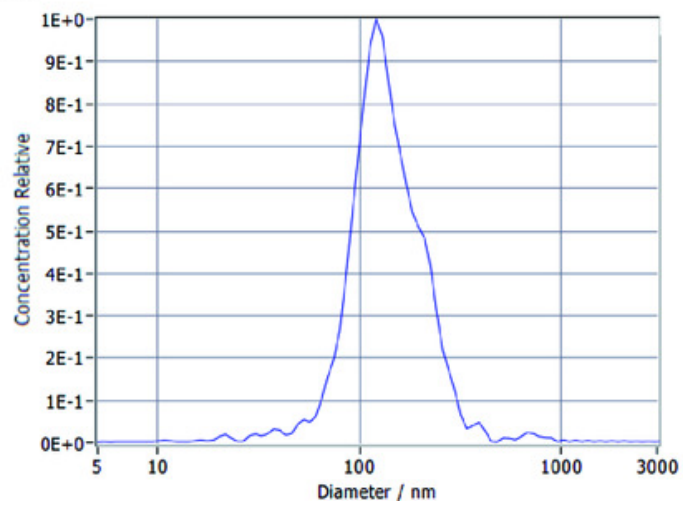

C

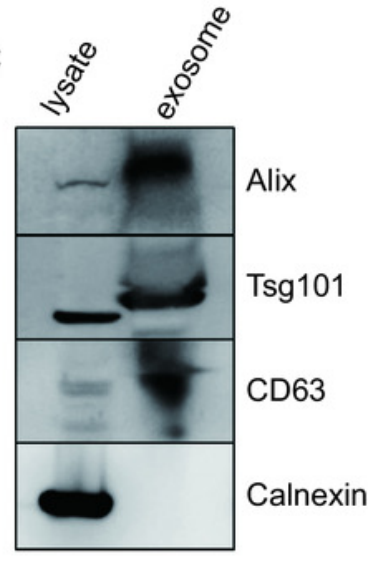


Figure 3

The different exosomal miRNA expression profiles and screening of AILI-related target genes.

(A) Heat map of DE-miRNAs. Red represents higher expression and blue represents lower expression. (B) Volcano plot of DE-miRNAs. The red and blue points represent significantly upregulated and downregulated miRNAs, respectively. (C) Venn diagram of target genes overlapped with DILI-related genes.

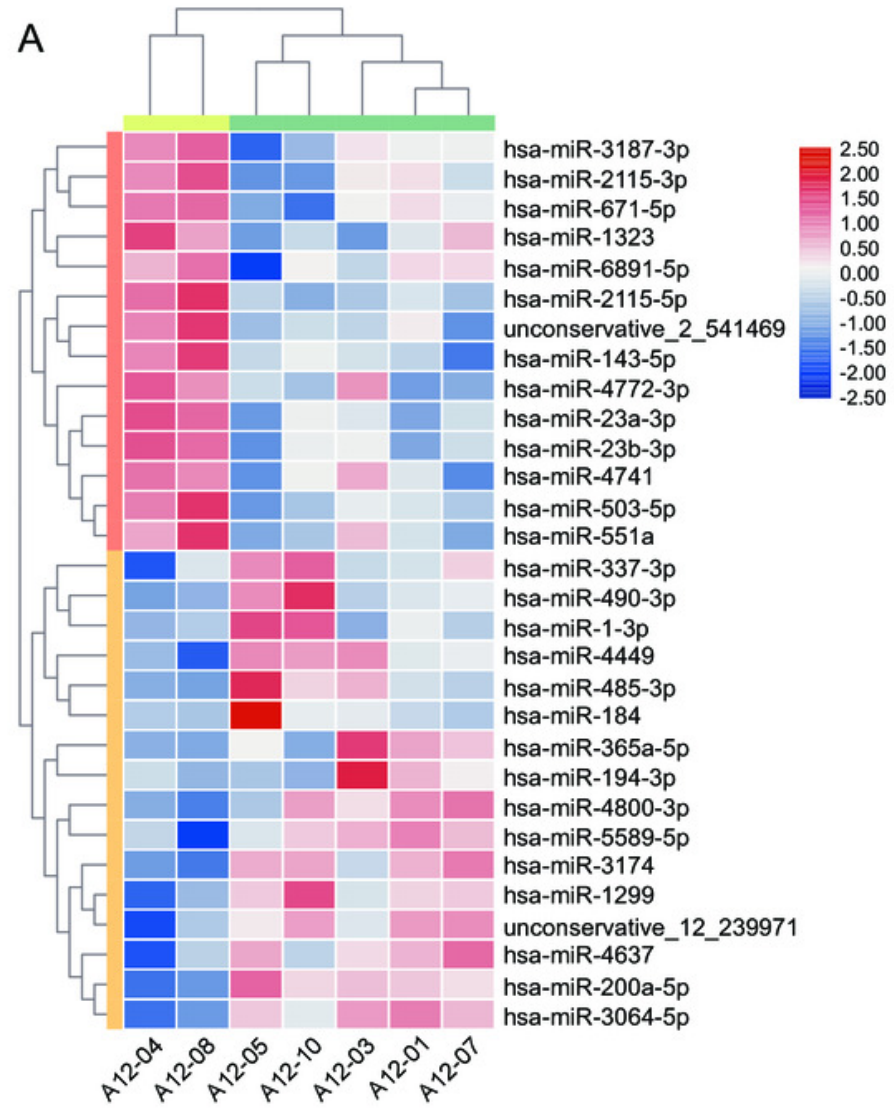

B

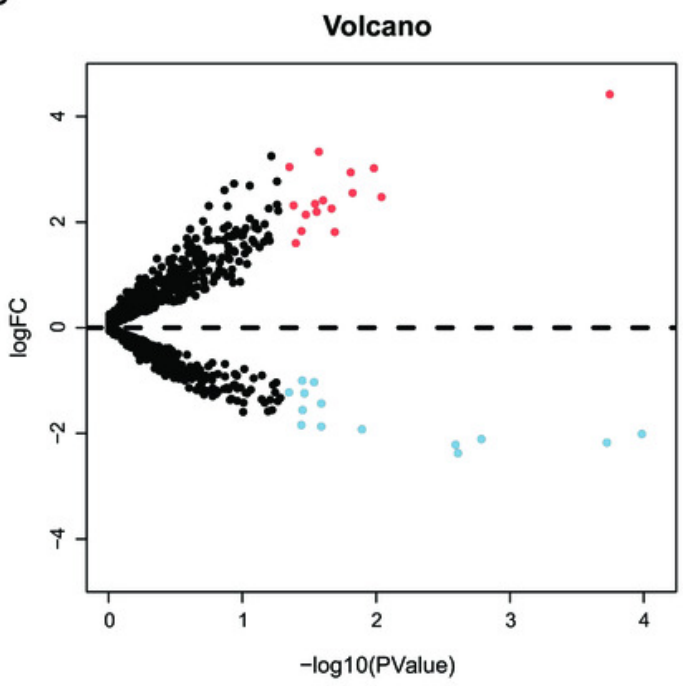

C

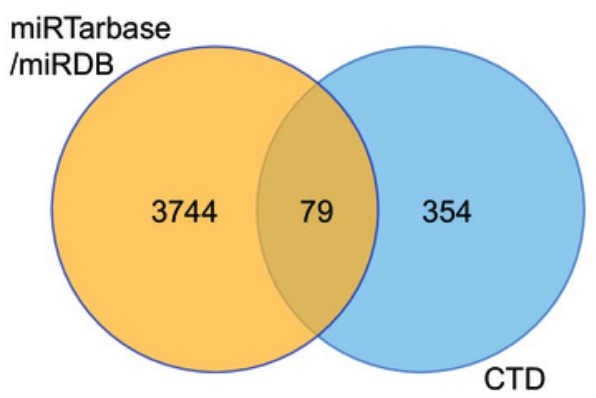


Figure 4

Bioinformatics analysis of AlLI-related target genes.

(A) GO annotation and KEGG pathway enrichment analysis for AlLI-related target genes of the upregulated miRNAs. (B) GO annotation and KEGG pathway enrichment analysis for AILIrelated target genes of the downregulated miRNAs. 
A
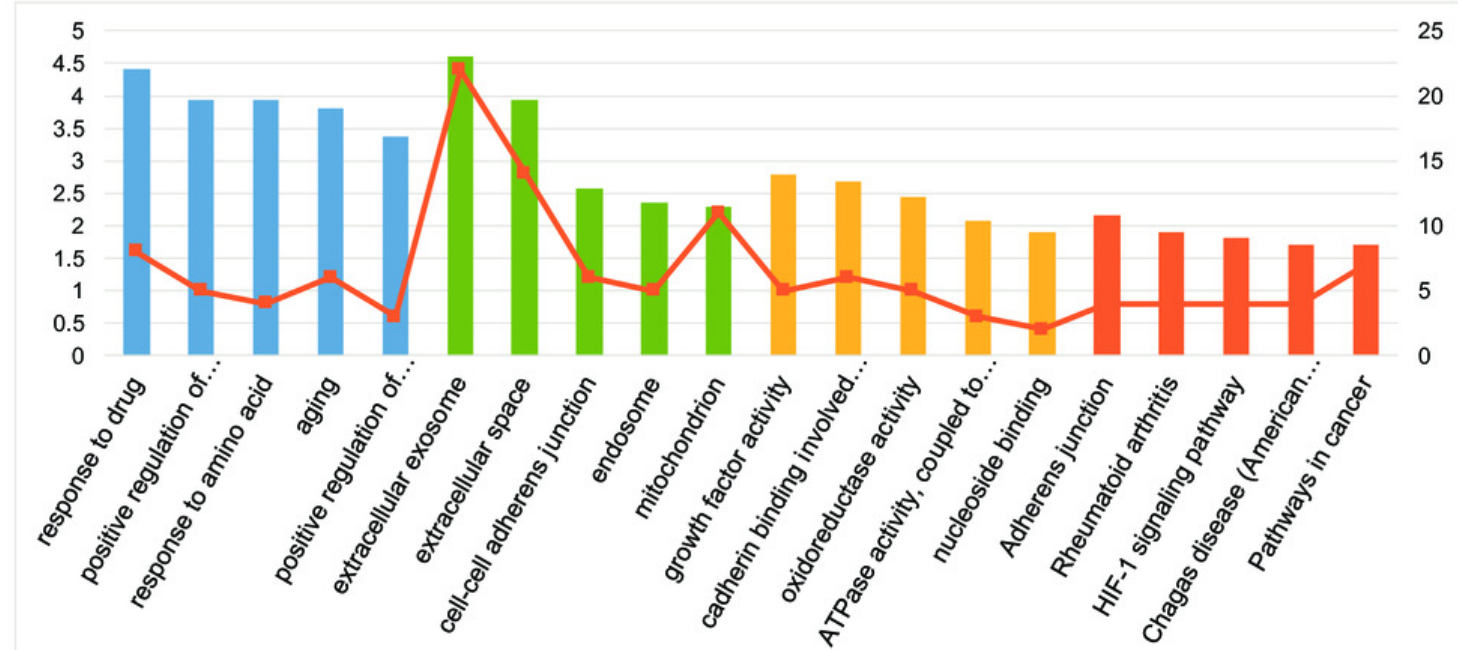

- -Log 10(Pvalue) $=$ Count

B

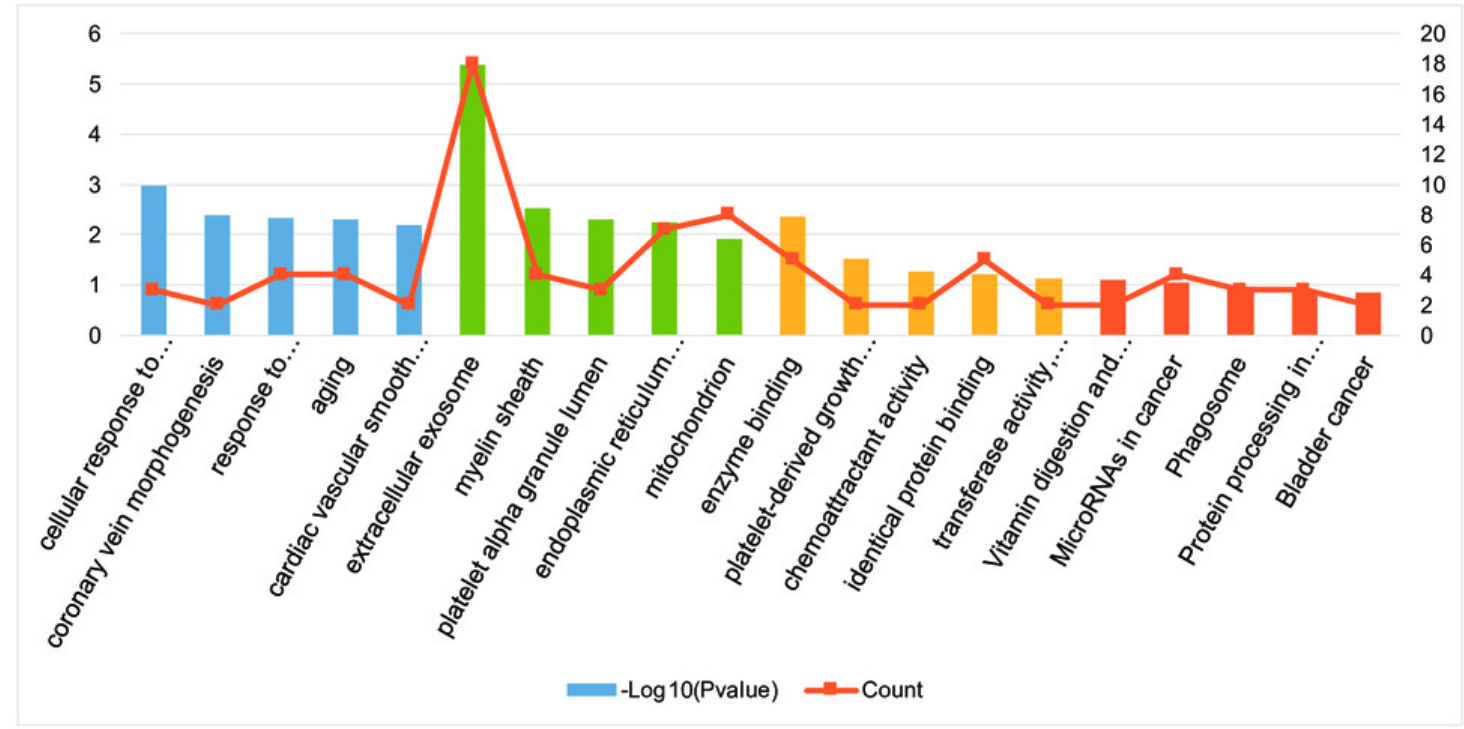




\section{Figure 5}

PPI network and miRNA-hub gene network.

(A) PPI network of AILI-related target genes. (B) miRNA-hub gene network. In the miRNA-hub gene network, the blue ellipses represent hub genes, the red diamonds represent upregulated miRNAs and the green diamonds represent downregulated miRNAs. 


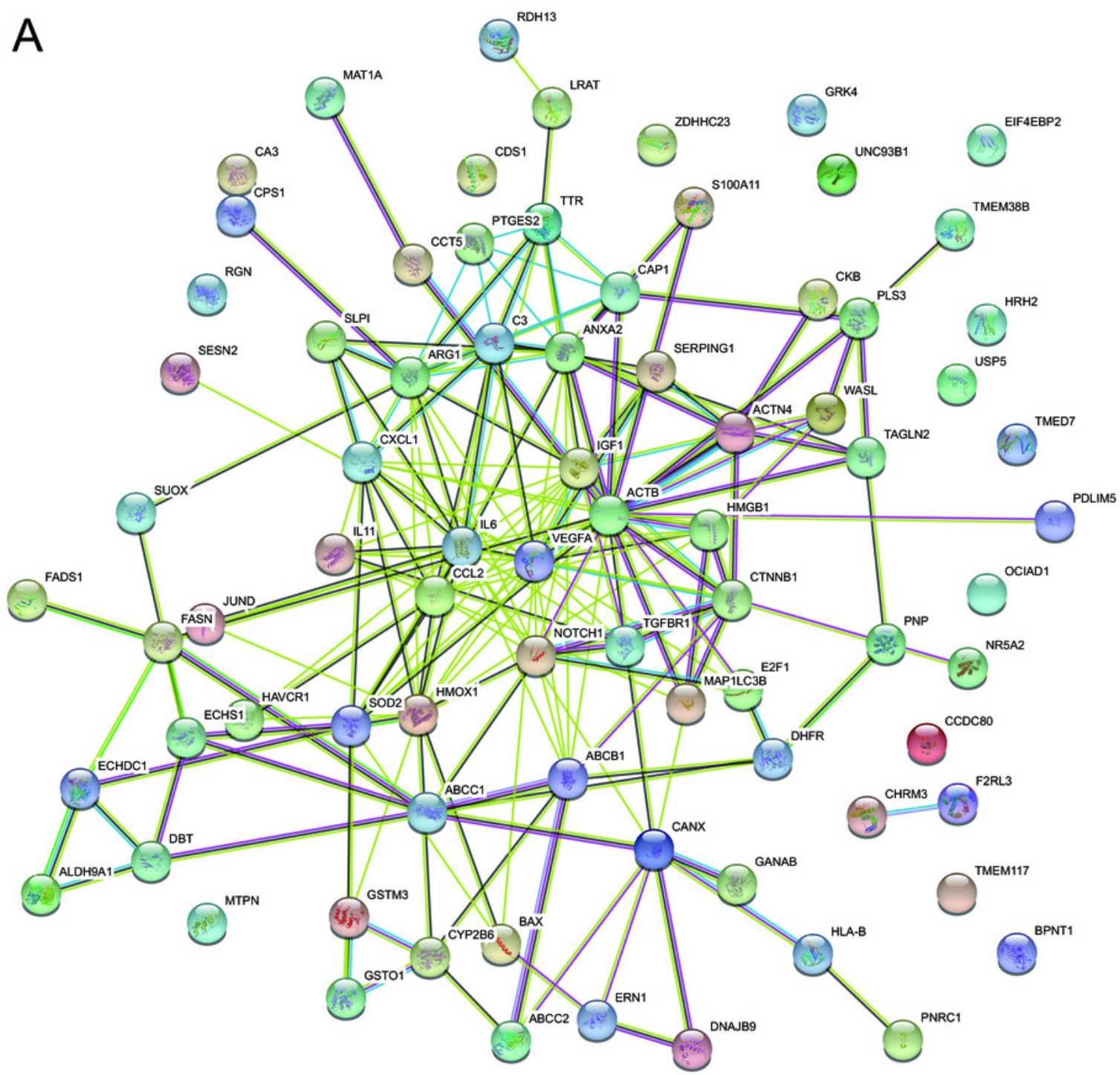

B

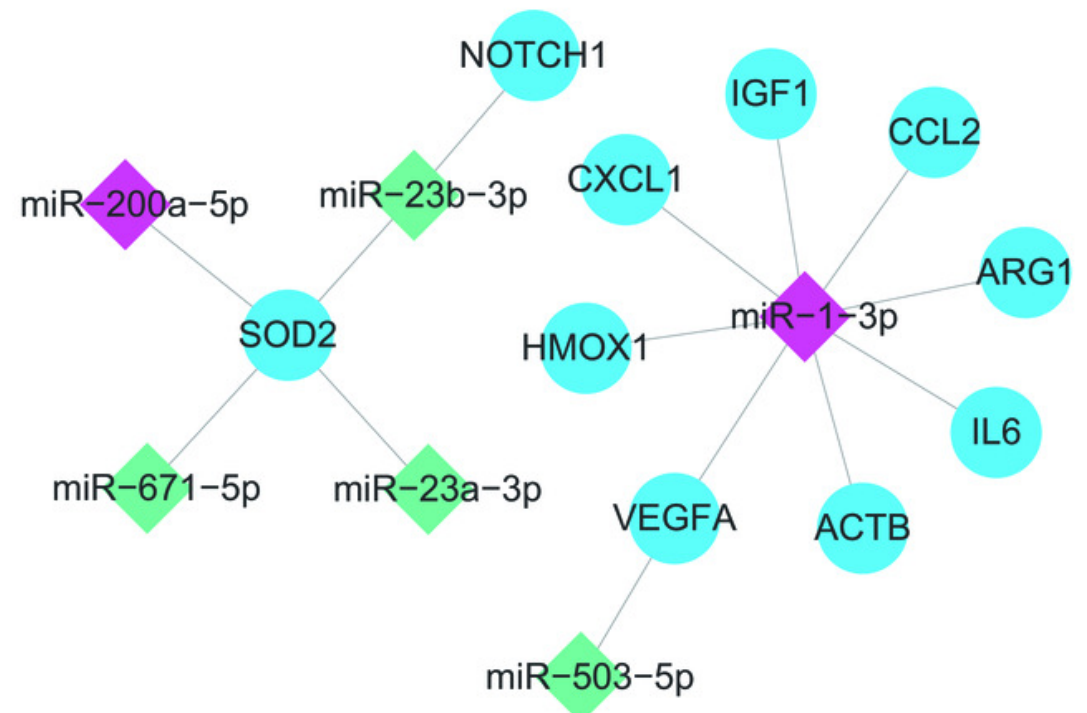




\section{Table $\mathbf{1}$ (on next page)}

Top 10 hub genes in the PPI network (ranked by MCC method). 


\begin{tabular}{lll}
\hline Rank & Name & Score \\
\hline 1 & IL6 & 8670 \\
2 & VEGFA & 8667 \\
3 & CCL2 & 8172 \\
4 & HMOX1 & 7938 \\
5 & IGF1 & 7626 \\
6 & ACTB & 6838 \\
7 & CXCL1 & 6726 \\
8 & SOD2 & 5052 \\
9 & NOTCH1 & 1808 \\
10 & ARG1 & 1748 \\
\hline
\end{tabular}

